\title{
Treatment of EGFR mutation-positive non-small cell lung cancer complicated by Trousseau syndrome with gefitinib followed by osimertinib: a case report
}

\author{
Yoshikane Nonagase $^{1}$, Masayuki Takeda ${ }^{1}$, Kaoru Tanaka ${ }^{1}$, Hidetoshi Hayashi ${ }^{1}$, \\ Tsutomu Iwasa ${ }^{1}$ and Kazuhiko Nakagawa ${ }^{1}$ \\ ${ }^{1}$ Department of Medical Oncology, Kindai University Faculty of Medicine, Osaka, Japan \\ Correspondence to: Masayuki Takeda, email: takedamasa2004@yahoo.co.jp \\ Keywords: lung cancer; gefitinib; osimertinib; Trousseau syndrome; EGFR mutation \\ Received: April 30, $2018 \quad$ Accepted: June 13, $2018 \quad$ Published: June 29, 2018 \\ Copyright: Nonagase et al. This is an open-access article distributed under the terms of the Creative Commons Attribution License \\ 3.0 (CC BY 3.0), which permits unrestricted use, distribution, and reproduction in any medium, provided the original author and \\ source are credited.
}

\section{ABSTRACT}

Malignant tumors can induce a hypercoagulable state known as Trousseau syndrome that increases the risk for venous thromboembolism including disabling cerebral infarction. Anticoagulant therapy without anticancer treatment is not effective for amelioration of this coagulation abnormality. Most patients with lung cancer positive for activating mutations of the epidermal growth factor receptor (EGFR) are sensitive to EGFR tyrosine kinase inhibitors (TKIs), but the efficacy and safety of EGFR-TKIs in such patients with a poor performance status (PS) due to Trousseau syndrome has been unclear. We here describe a patient with EGFR mutation-positive lung cancer who developed disabling cerebral infarction due to Trousseau syndrome. Administration of the EGFR-TKI gefitinib and anticoagulant therapy resulted in a partial tumor response and recovery from both the coagulation abnormality and the severe neurological symptoms. After the development of resistance to gefitinib, the EGFR-TKI osimertinib was safely administered until disease progression without recurrence of the coagulation abnormality. This case suggests that gefitinib followed by osimertinib may be a safe and effective treatment option for patients with EGFR mutation-positive lung cancer who experience disabling cerebral infarction due to Trousseau syndrome.

\section{INTRODUCTION}

Trousseau syndrome is a state of hypercoagulability associated with certain malignant tumors and can manifest as deep venous thrombosis or cerebral infarction [1]. Given that platinum-based chemotherapy increases the risk of thromboembolic complications, patients with lung cancer and severe cerebral infarction due to Trousseau syndrome are often considered to be ineligible for such treatment [2]. Epidermal growth factor receptor (EGFR) tyrosine kinase inhibitors (TKIs) are now a standard first-line therapy for patients with advanced non-small cell lung cancer (NSCLC) positive for activating EGFR mutations, with these drugs also being efficacious and tolerable even in individuals with a poor Eastern Cooperative Oncology Group (ECOG) performance status
(PS) associated with disease progression [3]. We now report a case of acute cerebral infarction due to Trousseau syndrome in a patient who was recently diagnosed with lung cancer positive for an activating EGFR mutation and who was subsequently treated safely with the EGFR-TKIs gefitinib and osimertinib.

\section{CASE REPORT}

A 53-year-old man with no medical history of arrhythmia, diabetes mellitus, coagulation disorder, or stroke consulted our hospital complaining of back pain. Contrast-enhanced computed tomography (CT) showed a 21-mm-diameter nodule in the lower left lung as well as multiple liver and bone metastases (Figure 1A), but no abnormalities in the brain. Percutaneous needle biopsy of 
the liver led to a diagnosis of adenocarcinoma of the lung (T1bN3M1c, cStage IVb) positive for an exon 19 deletion of the EGFR gene.

Before initiation of treatment with gefitinib, the patient was admitted to the hospital because of a disturbance of consciousness and malaise. His ECOG PS was 4. A brain CT scan again showed no abnormalities, whereas laboratory tests revealed a decreased platelet count of 59,000/ $\mu 1$ (normal range, 158,000 to $348,000 / \mu \mathrm{l}$ ), an increased prothrombin time/international normalized ratio (PT-INR) of 1.35 (normal range, 0.90 to 1.10 ), and an increased fibrin degradation product level of $174.3 \mu \mathrm{g} / \mathrm{ml}$ (normal range, 0 to $8 \mu \mathrm{g} / \mathrm{ml}$ ), suggestive of cancer-associated disseminated intravascular coagulation. Anticoagulant therapy with thrombomodulin alfa $(380 \mathrm{U} / \mathrm{kg})$ was initiated. Transthoracic echocardiography revealed no findings of valvular disease or intracardiac thrombus. On his second day in hospital, the patient was started on gefitinib at $250 \mathrm{mg} /$ day, given that this drug has been shown to be safe and effective in EGFR mutation-positive
NSCLC patients with a poor PS [3]. After 2 days of treatment with gefitinib, the patient presented with right hemiplegia, aphasia, and cognitive dysfunction. Diffusionweighted magnetic resonance imaging (DW-MRI) revealed multiple acute cerebral infarctions (Figure 2A) and the patient was diagnosed with Trousseau syndrome. $\mathrm{He}$ received intravenous unfractionated heparin with a target activated partial thromboplastin time of 40 to $60 \mathrm{~s}$ (normal range, 26 to $35 \mathrm{~s}$ ) for 7 days, and he was treated with warfarin to maintain his PT-INR between 1.5 and 2.5. The hemiplegia, aphasia, and cognitive dysfunction were gradually ameliorated. After 11 days of treatment with gefitinib, laboratory data showed an improvement in the platelet count from 48,000 to $480,000 / \mu 1$, CT revealed a partial tumor response according to RECIST criteria (Figure 1B), and DW-MRI detected no further cerebral infarction (Figure 2B). After treatment with gefitinib for 7 months, the patient showed disease progression with regard to the liver metastases (Figure 1C) without worsening of his coagulant profile. Rebiopsy of liver metastases to examine status for the T790M resistance

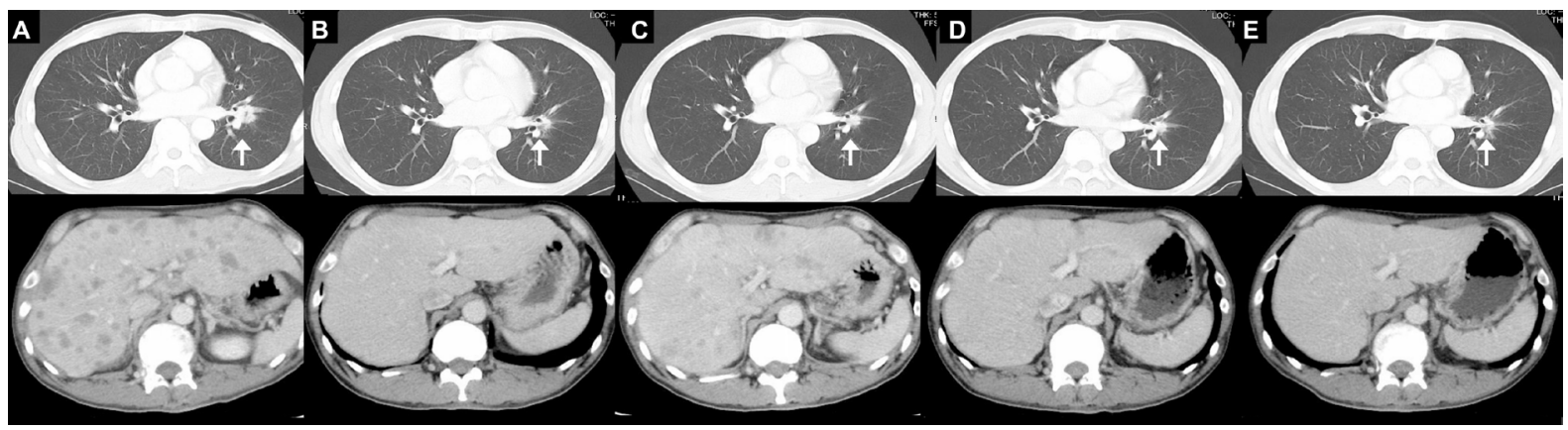

Figure 1: Contrast-enhanced CT scans of the lungs and liver. Images were acquired before treatment with gefitinib (A), 11 days after the onset of gefitinib treatment (B), 7 months after treatment onset, when disease progression was apparent for the liver metastases $(\mathbf{C})$, 1 month after the onset of treatment with osimertinib (D), and after 3 months of treatment with osimertinib, when leptomeningeal metastasis had occurred (E). Arrows indicate primary lung lesion.

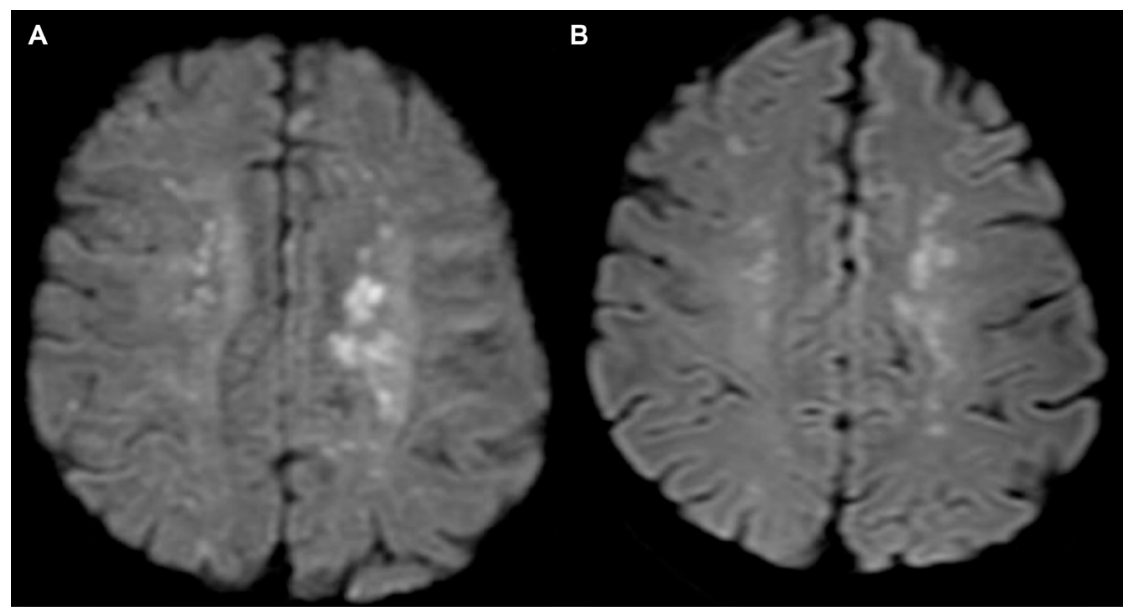

Figure 2: DW-MRI imaging of the brain. Images were acquired after acute cerebral infarction (A) and 11 days after the onset of treatment with gefitinib together with anticoagulant therapy $(\mathbf{B})$. 
mutation of $E G F R$ was difficult because of the small lesion size and the continuation of anticoagulant therapy. We were able to detect the T790M mutation by analysis of plasma cell-free tumor DNA with the cobas EGFR Mutation Test (Roche), however, allowing a switch to osimertinib only 5 days after disease progression during gefitinib treatment. Exacerbation of the coagulation abnormality did not recur during treatment with osimertinib. Although shrinkage of the primary lung lesion and liver metastases was maintained during treatment with osimertinib (Figure 1D, 1E), the patient showed leptomeningeal disease progression 3 months after initiation of this therapy. No further chemotherapy was administered because of his poor ECOG PS associated with disease progression, and he died 1 month after discontinuation of osimertinib.

\section{DISCUSSION}

There is no established therapy for patients with EGFR mutation-positive NSCLC and Trousseau syndrome who present with severe neurological symptoms. In the present case, consecutive treatment with the EGFR-TKIs gefitinib and osimertinib was safe and effective until disease progression. To our knowledge, this is the first report of such treatment leading to a good response and recovery from neurological symptoms without recurrence of the coagulation abnormality in a patient with EGFR mutation-positive NSCLC and Trousseau syndrome.

Anticoagulant therapy alone for cancerassociated thromboembolism is often not effective without concomitant anticancer treatment [4]. Given that malignant cells produce and release procoagulant factors including mucins observed in the present case, amelioration of the coagulation abnormality might require a reduction in tumor volume $[5,6]$. Conventional cytotoxic chemotherapy is not routinely administered to patients with a poor ECOG PS linked to disease progression, and platinum-based chemotherapy can increase the risk of thromboembolism [2]. In contrast, EGFR-TKIs can be administered safely even to patients with a poor ECOG PS, and they are associated with a higher tumor response rate and shorter time to response compared with chemotherapy [3, 7]. EGFR-TKIs may thus be a better treatment option than chemotherapy for patients with EGFR mutation-positive NSCLC complicated by Trousseau syndrome.

In the present case, exacerbation of the coagulation abnormality did not recur during treatment with gefitinib followed by osimertinib, possibly as a result of the associated marked reduction in tumor volume. The risk for recurrence of venous thromboembolism in cancer patients is thought to be higher for those with more extensive disease [8]. In our case, the tumor volume during gefitinib and osimertinib treatment as well as after subsequent disease progression was much smaller than that before initiation of anticancer therapy. The pronounced reduction in tumor burden achieved with gefitinib and osimertinib might thus have contributed to lowering of the risk for recurrence of venous thromboembolism. We cannot exclude the possibility that the combination of chemotherapy and anticoagulant therapy may have been responsible for the lack of recurrence of thromboembolic events. The recurrence rate for cerebral infarction in patients with cancer was found to be $16 \%$ within 6 months, whereas that in patients without cancer receiving anticoagulant therapy was 5.9 to $10.3 \%$ within the 1 st year $[9,10]$.

A case of NSCLC associated with Trousseau syndrome was previously found to be controlled with gefitinib followed by erlotinib and then by carboplatin plus pemetrexed [11]. However, the coagulation abnormality recurred several months after initiation of each regimen, presumably because no substantial tumor shrinkage was obtained. Moreover, osimertinib had not been approved at the time of this previous case. For the present case, we were able to switch from gefitinib to osimertinib promptly as a result of the rapid determination of T790M mutation status by liquid biopsy. The results of a recent phase III trial of osimertinib in comparison with gefitinib in previously untreated patients with EGFR mutationpositive advanced NSCLC suggest the possibility that osimertinib may be a treatment option for chemonaïve patients with EGFR-mutated NSCLC and Trousseau syndrome [12].

In conclusion, gefitinib followed by osimertinib may be a safe and effective treatment option for EGFR mutation-positive NSCLC patients with a poor ECOG PS due to Trousseau syndrome.

\section{CONFLICTS OF INTEREST}

K.N. has received honoraria from AstraZeneca Pharmaceutical Co. Ltd. The remaining authors declare no conflict of interest.

\section{REFERENCES}

1. Varki A. Trousseau's syndrome: multiple definitions and multiple mechanisms. Blood. 2007; 110:1723-9.

2. Kim ES, Baran AM, Mondo EL, Rodgers TD, Nielsen GC, Dougherty DW, Pandya KJ, Rich DQ, van Wijngaarden E. Risk of thromboembolism in cisplatin versus carboplatintreated patients with lung cancer. PLoS One. 2017; 12:e0189410.

3. Inoue A, Kobayashi K, Usui K, Maemondo M, Okinaga S, Mikami I, Ando M, Yamazaki K, Saijo Y, Gemma A, Miyazawa H, Tanaka T, Ikebuchi K, et al. First-line gefitinib for patients with advanced non-small-cell lung cancer harboring epidermal growth factor receptor mutations 
without indication for chemotherapy. J Clin Oncol. 2009; 27:1394-400.

4. Kuwahata S, Takenaka T, Yasuda S, Sakoda T, Taniyama H, Nagata Y, Iwakawa J, Matsumoto S, Seto T, Takenoyama M, Abe S, Ohishi M. A case of anticoagulant treatmentresistant Trousseau syndrome controlled by treatment of the underlying lung adenocarcinoma: Utility of monitoring D-dimer levels. Journal of Cardiology Cases. 2017; 15:65-9.

5. Caine GJ, Stonelake PS, Lip GY, Kehoe ST. The hypercoagulable state of malignancy: pathogenesis and current debate. Neoplasia. 2002; 4:465-73.

6. Wahrenbrock M, Borsig L, Le D, Varki N, Varki A. Selectinmucin interactions as a probable molecular explanation for the association of Trousseau syndrome with mucinous adenocarcinomas. The Journal of Clinical Investigation. 2003; 112:853-62.

7. Takeda M, Okamoto I, Nakagawa K. Survival outcome assessed according to tumor response and shrinkage pattern in patients with EGFR mutation-positive non-small-cell lung cancer treated with gefitinib or erlotinib. J Thorac Oncol. 2014; 9:200-4.

8. Prandoni P, Lensing AW, Piccioli A, Bernardi E, Simioni P, Girolami B, Marchiori A, Sabbion P, Prins MH, Noventa F, Girolami A. Recurrent venous thromboembolism and bleeding complications during anticoagulant treatment in patients with cancer and venous thrombosis. Blood. 2002; 100: 3484-8.
9. Soda T, Nakayasu H, Maeda M, Kusumi M, Kowa H, Awaki E, Saito J, Nakashima K. Stroke recurrence within the first year following cerebral infarction-Tottori University Lacunar Infarction Prognosis Study (TULIPS). Acta Neurol Scand. 2004; 110:343-9.

10. Navi BB, Singer S, Merkler AE, Cheng NT, Stone JB, Kamel H, Iadecola C, Elkind MS, DeAngelis LM. Recurrent thromboembolic events after ischemic stroke in patients with cancer. Neurology. 2014; 83:26-33.

11. Masubuchi H, Maeno T, Uchida M, Kono S, Suzuki M, Takemura M, Yamaguchi A, Yamaguchi K, Kanbe M, Kitahara S, Hara K, Hara S, Aoki N, et al. A case of Trousseau syndrome caused by pulmonary adenocarcinoma that was controlled for one year and 10 months with thrombosis treatment using an EGFR tyrosine kinase inhibitor and chemotherapy. Respir Med Case Rep. 2015; 15:101-5.

12. Soria JC, Ohe Y, Vansteenkiste J, Reungwetwattana T, Chewaskulyong B, Lee KH, Dechaphunkul A, Imamura F, Nogami N, Kurata T, Okamoto I, Zhou C, Cho BC, et al. Osimertinib in untreated EGFR-mutated advanced nonsmall-cell lung cancer. N Engl J Med. 2018; 378:113-25. 